\title{
DESHIDRATACIÓN OSMÓTICA DE PULPA DE TAMARINDO (Tamarindus indica L.): INFLUENCIA DE LA TEMPERATURA Y LA CONCENTRACIÓN
}

\section{OSMOTIC DEHYDRATION TAMARIND PULP (Tamarindus indica L. ): INFLUENCE OF TEMPERATURE AND CONCENTRATION}

\author{
Diofanor Acevedo ${ }^{1}$, Diego Tirado², Luis Guzmán ${ }^{3}$
}

\begin{abstract}
${ }^{1}$ Ingeniero de Alimentos. Ph.D. Docente Universidad de Cartagena, Programa Ingeniería de Alimentos, Universidad de Cartagena, Facultad de Ingeniería, Campus Piedra de Bolívar, Cartagena-Bolívar, Colombia, e-mail: diofanor3000@gmail.com; ${ }^{2}$ Ingeniero de Alimentos, Joven Investigador e Innovador COLCIENCIAS, Universidad de Cartagena, Programa Ingeniería de Alimentos, Universidad de Cartagena, Facultad de Ingeniería, Campus de Piedra de Bolívar, Cartagena-Bolívar, Colombia, email: dtiradoa@unicartagena.edu.co; ${ }^{3}$ Ingeniero de Alimentos. Esp. Docente Universidad de Cartagena, Programa Ingeniería de Alimentos, Universidad de Cartagena, Facultad de Ingeniería, Campus Piedra de Bolívar, Cartagena-Bolívar, Colombia. e-mail: lguzmanc1@unicartagena.edu.co
\end{abstract}

Rev. U.D.C.A Act. \& Div. Cient. 17(1): 123-130, Enero-Junio, 2013

\section{RESUMEN}

La deshidratación osmótica en la industria de alimentos mejora la calidad de productos hortofrutícolas y aumenta su estabilidad. En el presente trabajo, se evaluó la cinética de deshidratación osmótica en pulpa de tamarindo (Tamarindus indica L.), a partir del índice de efectividad y se estudió la influencia de la temperatura y la concentración de la solución sobre el proceso. Para ello, se determinó experimentalmente la pérdida de agua, pérdida de peso y ganancia de sólidos durante la deshidratación osmótica de la pulpa, en soluciones hipertónicas de sacarosa, con 30, 40 y 60 Brix y temperaturas de 29 y $50^{\circ} \mathrm{C}$. Como resultado, se obtuvo que a mayor concentración y temperatura de la solución, se produzcan mayores pérdidas de agua $(53,9 \%)$, peso $(53,9 \%)$ y ganancia de sólidos (0,008\%).

Palabras clave: Productos hortofrutícolas, índice de efectividad, pérdida de agua (WL), pérdida de peso (WR), ganancia de sólidos (GS).

\section{SUMMARY}

Industrial osmotic food dehydration improves the quality of horticultural products and increases its stability. In this study the kinetics of osmotic dehydration of tamarind pulp (Tamarindus indica L.) was evaluated based on the index of effectiveness and the influence of temperature and solution concentration on the process was determined. The experimentally water loss and weight loss and the gain of solids during osmotic dehydration within hypertonic sucrose solutions of 30, 40 and $60^{\circ}$ Brix, and temperatures of 29 and $50^{\circ} \mathrm{C}$ was studied. The results showed that the higher the solution concentration and temperature, a higher water loss $(53.9 \%)$, weight $(53.9 \%)$ and solid gain $(0,008 \%)$ was produced.

Key words: Horticultural products, water loss (WL), weight loss (WR), solid gain (SG), rate of effectiveness.

\section{INTRODUCCIÓN}

El tamarindo (Tamarindus indica L.) es originario del continente Africano y fue introducido al Continente Americano por los españoles. Pertenece a la familia de las leguminosas (Fabaceae). Es un frutal altamente rústico, ya que puede prosperar en suelos pobres o marginados, con poco o nada de riego y cuidados mínimos con relación a otros frutales tropicales (Orozco-Santos, 2001).

El fruto es una vaina curvada de 12 a $15 \mathrm{~cm}$ de longitud, del cual, la pulpa constituye de 30 a $55 \%$, la cáscara y la fibra de 11 a $30 \%$ y las semillas de color marrón, de 33 a $44 \%$. La cosecha, se realiza cuando el fruto alcanza su madurez fisiológica, que sucede cuando se deshidrata; pierde peso y adquiere un color gris pardo o marrón y un sonido hue- 
co, cuando chocan entre sí. Además, la cáscara del fruto se torna quebradiza, cuando se presiona ligeramente con los dedos (Orozco-Santos, 2001).

El objetivo principal de la explotación del cultivo del tamarindo es la producción de pulpa para su uso en la elaboración de dulces, jugos, jaleas, pulpas, refrescos embotellados, helados y salsas (Ahmed et al. 2007).

Actualmente, en el sector agroindustrial, se emplean diversos tipos de procesos tecnológicos de conservación y entre los más utilizados, se encuentran los procesos de deshidratación, que resultan ser una técnica efectiva, para lograr productos estables, con características sensoriales y nutritivas, posibilitando la obtención de un producto para el consumo, con cierto parecido a él mismo en estado fresco y productos mínimamente procesados (García et al. 2013). En el caso específico de las frutas, los métodos de conservación más recomendados son: el método de deshidratación osmótica (DO) y el método de deshidratación por flujo de aire caliente (DAC). La DO, se utiliza ampliamente para eliminar parcialmente el agua de los tejidos vegetales y la obtención de un aumento significativo de su tiempo de vida útil, mediante inmersión en una solución hipertónica (García et al. 2013; Cataldo et al. 2011).

La DO es una técnica de remoción de agua, que se caracteriza por presentar dos etapas: una dinámica y otra de equilibrio. En la etapa dinámica, las velocidades de transferencia de materia disminuyen hasta que se llega al equilibrio. El proceso osmótico termina cuando se alcanza este equilibrio, es decir, cuando la velocidad neta de transporte de materia es cero (Della Rocca \& Mascheroni, 2011). En la difusión del agua del alimento a la solución, la fuerza impulsora requerida para el flujo del agua es la diferencia de potencial químico entre la disolución y el fluido intracelular. Si la membrana celular es perfectamente semipermeable, el soluto es incapaz de difundir hacia el interior de las células; sin embargo, en los alimentos es difícil tener membranas perfectamente semipermeables, debido a su compleja estructura interna, produciéndose alguna difusión del soluto al alimento y viceversa, además de una lixiviación de pequeñas cantidades de compuestos solubles del alimento hacia la solución, que pueden afectar las características sensoriales y nutritivas del producto (Dermesonlouoglou et al. 2008; Toğrul \& Íspir, 2008). Por consiguiente, el fenómeno presenta dos procesos simultáneos en contracorriente: el movimiento de agua desde el alimento hacia la solución hipertónica, en el que se pueden arrastrar algunos componentes disueltos del alimento junto con el agua extraída y, por otro lado, la impregnación del alimento con los solutos que provienen de la solución (Toğrul \& Íspir, 2008).

La cinética de los procesos de DO, se evalúan, por lo gene- ral, en términos de pérdida de agua, pérdida de peso y ganancia de sólidos y depende, principalmente, de las características de la materia prima y de las condiciones del proceso, tales como composición y concentración del soluto osmótico, temperatura, tiempo de inmersión, nivel de agitación y características específicas del alimento (tamaño y forma) y la relación solución alimento (Dermesonlouoglou et al. 2008; Silva et al. 2012).

El proceso de DO, se puede evaluar en términos de parámetros cinéticos, mediante el empleo del índice de efectividad. Éste, se obtiene mediante la relación entre la pérdida de agua y la ganancia de sólidos (WL/SG). Es un índice empleado para evaluar la efectividad del proceso de osmodeshidratación y es considerado como un parámetro de calidad. Valores altos de esta relación indican una mejor condición del proceso (Ayala et al. 2010). En el presente trabajo, se evaluó la cinética de deshidratación osmótica en pulpa de tamarindo ( $T$. indica L.) y se estudió la influencia de la temperatura y la concentración de la solución sobre el proceso.

\section{MATERIALES Y MÉTODOS}

Material vegetal. La pulpa de tamarindo con $12^{\circ}$ Brix, se obtuvo retirando la cáscara y dejando la semilla.

Deshidratación osmótica y diseño experimental. Se empleó un diseño experimental factorial general de dos factores: concentración de sacarosa y temperatura; con tres niveles para la sacarosa: 30,40 y $60^{\circ}$ Brix; y dos para la temperatura: 29 y $50^{\circ} \mathrm{C}$. Se realizaron 18 corridas experimentales, siguiendo un orden aleatorio.

Se trabajó con una masa de pulpa de $100 \mathrm{~g}$ en forma cilíndrica y $1000 \mathrm{~g}$ de solución osmótica, teniendo así una relación 1:10. Los ensayos fueron realizados en un baño termostático marca FERCA, modelo TT400, con agitación lineal, para evitar el efecto de encostramiento del azúcar en la superficie de las muestras y acelerar el proceso de deshidratación (Ayala et al. 2010).

Para evaluar las variables de respuesta, pérdida de peso (WR), pérdida de agua (WL) y ganancia de sólidos solubles (SG), se tomaron muestras de pulpa por triplicado a diferentes tiempos de osmodeshidratación (1, 2, 3, 4, 5, 6, 7, 8, 9 y 10 horas).

Una vez extraídas de la solución osmodeshidratante (SO), las muestras se lavaron en agua destilada, para eliminar la solución remanente y se secaron con papel absorbente. Las muestras, se pesaron antes y después de la deshidratación, en una balanza analítica con sensibilidad de 0,001g. Los sólidos solubles, se determinaron a la solución osmodeshidratante por el método refractométrico, siguiendo la Norma 
Técnica Colombiana 440 (1971). El contenido de humedad, se determinó a $105^{\circ} \mathrm{C}$ hasta peso constante (AOAC, 2005). Las medidas experimentales, se realizaron por triplicado para cada tratamiento osmótico.

El cálculo de los parámetros de pérdida de agua (WL), pérdida de peso (WR) y ganancia de sólidos (SG), se calcularon mediante la adaptación de las ecuaciones de Agnelli et al. (2005), expresadas en $\mathrm{g} / 100 \mathrm{~g}$ de producto fresco:

$$
\begin{aligned}
& W L=\frac{\left(M_{0} \times H_{0}\right)-\left(M_{t} \times H_{t}\right)}{M_{0}} \times 100 \\
& W R=\frac{\left(M_{0}-M_{t}\right)}{M_{0}} \times 100 \\
& S G=\frac{\left(M_{t} \times S_{t}\right)-\left(M_{0} \times S_{0}\right)}{M_{0}} \times 100
\end{aligned}
$$

Dónde:

$M_{\circ}=$ Pesos Inicial de la pulpa

$M_{\mathrm{t}}=$ pesos final de la pulpa

$\mathrm{H}_{\mathrm{o}}=$ Humedad inicial de la pulpa

$\mathrm{H}_{\mathrm{t}}=$ Humedad final de la pulpa

$\mathrm{S}_{\mathrm{o}}=$ Sólidos iniciales de la pulpa

$\mathrm{S}_{\mathrm{t}}=$ Sólidos finales de la pulpa

Índice de efectividad. Se calculó como el cociente entre la pérdida de agua y la ganancia de sólidos (WL/SG), ilustrados en las Ecuaciones 1 y 3 , respectivamente, en los tiempos de osmodeshidratación (1, 2, 3, 4, 5, 6, 7, 8, 9 y 10 horas).

Análisis estadístico. Para el análisis estadístico, se utilizó el programa es Statgraphics Centurion XVI.I, con análisis de varianza y correlación entre variables.

\section{RESULTADOS Y DISCUSIÓN}

En la figura 1, se aprecia la pérdida de agua de la pulpa de tamarindo a medida que transcurre el tiempo de deshidratación. Los cambios más significativos en la pérdida de agua (WL), se presentaron durante las primeras cuatro horas de la deshidratación osmótica, para la mayor temperatura de proceso $\left(50^{\circ} \mathrm{C}\right)$ y de cinco horas, para la de menor $\left(30^{\circ} \mathrm{C}\right)$. La pérdida de agua de la pulpa de tamarindo alcanzó valores de $52,8,52,9$ y $53,5 \%$, para concentraciones de solución del 30,40 y $60 \%$, respectivamente, a $50{ }^{\circ} \mathrm{C}$, durante las cuatro primeras horas y de $48,9,49,6$ y $51,8 \%$, para las concentraciones de solución del 30, 40 y $60 \%$, respectivamente, a $30^{\circ} \mathrm{C}$, durante las cuatro primeras horas. La figura 1 de la cinética de la deshidratación osmótica es similar a las publi- cadas por varios investigadores (Kowalska \& Lenart, 2001; Spiazzi \& Mascheroni, 1997; Lazarides et al. 1995; Palou et al. 1994; Park et al. 2002). Las mayores tasas de pérdida de agua en los ensayos realizados, se muestran al inicio del proceso, como consecuencia de una mayor fuerza osmótica de deshidratación existente entre el alimento y la solución hipertónica, como indican Salvatori et al. (1999). Moreira \& Xidieh (2004) explican que el aumento de la concentración y la temperatura de la solución osmótica favorecen la pérdida de agua, debido al mayor gradiente de presión osmótica en la interface producto/solución, dando una mayor velocidad de transferencia de masa. Estos resultados concuerdan con los reportados por Della Roca \& Mascheroni (2011), los cuales, determinaron las condiciones óptimas de deshidratación osmótica de papas, hallaron que la mayor pérdida de peso se presentó entre 1,5 a 2 horas de proceso y que esta resulta ser superior a medida que aumenta la temperatura de proceso y la concentración de la solución osmodeshidratante.

En la figura 2, se aprecia cómo la pérdida de peso de la pulpa es mayor a medida que aumenta la temperatura del proceso y la concentración de sacarosa en la solución hipertónica. Luego de 4 - 5 horas de deshidratación osmótica, se tienen las mayores pérdidas de peso, las que corresponden a una mayor pérdida de agua y, por lo tanto, una menor concentración de sólidos solubles en la solución (Della Rocca \& Mascheroni, 2011).

En la figura 3, se puede observar un aumento en la ganancia de sólidos, (GS) con el incremento de la temperatura y de la concentración de la solución osmodeshidratante. Cerca de las siete horas del proceso, se alcanza un estado de cuasi equilibrio, en donde la ganancia de sólidos sigue igual hasta finalizar la osmodeshidratación. Si se observa en la figura $3 a$, luego de las seis horas, la ganancia de sólidos decae, lo cual, se puede atribuir al fenómeno a que se lleva a cabo una impregnación del material con solutos provenientes de la solución. A bajas concentraciones, la SG es mayor que la WL y, a medida que aumenta la concentración de la solución osmótica, la SG alcanza su máximo valor antes de disminuir y volverse inferior al WL. Niveles elevados de SG no permiten una elevada WL, a causa de la formación de una capa superficial de azúcar. Si se desea limitar la impregnación es conveniente usar altas concentraciones de soluto y tiempos de deshidratación osmótica cortos (Della Roca \& Mascheroni, 2011).

Este fenómeno ocurrió en otros trabajos, como los de Della Roca \& Mascheroni (2011), quienes durante la deshidratación osmótica de papas, observaron que a bajas concentraciones de sacarosa y sal disminuye la ganancia de sólidos, debido, probablemente, a la formación de una capa superficial sobre el producto que impide el ingreso de sólidos dentro del mismo. Otros autores (Giraldo et al. 2004; Mújica-Paz 


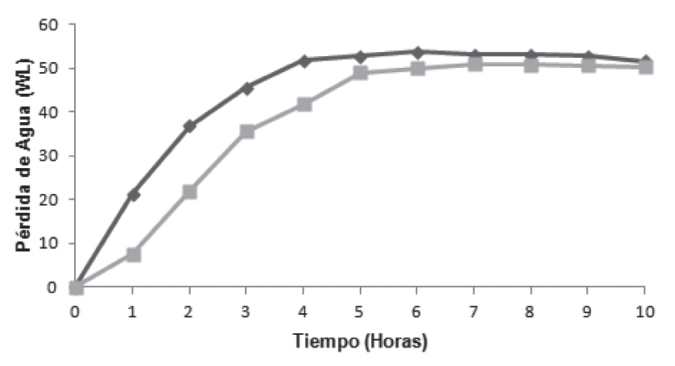

a)

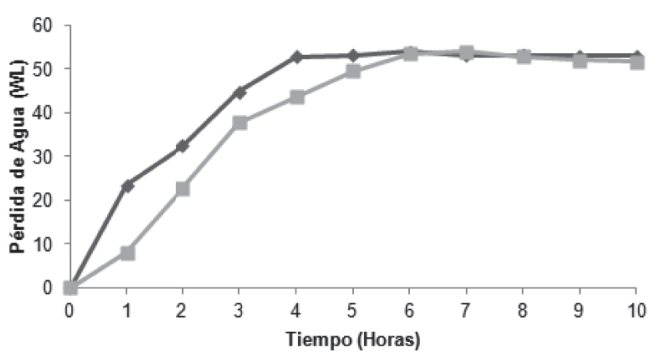

b)

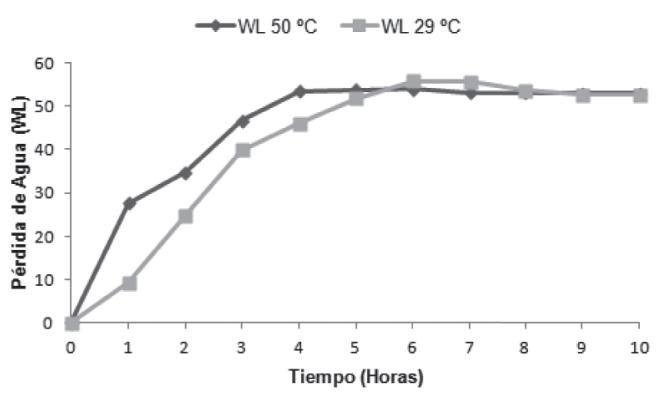

c)

Figura 1. Pérdida de agua (WL) a diferente concentración y temperatura: a) $\left.\left.30^{\circ} \mathrm{Brix}, \mathrm{b}\right) 40^{\circ} \mathrm{Brix}, \mathrm{c}\right) 60^{\circ} \mathrm{Brix}$.

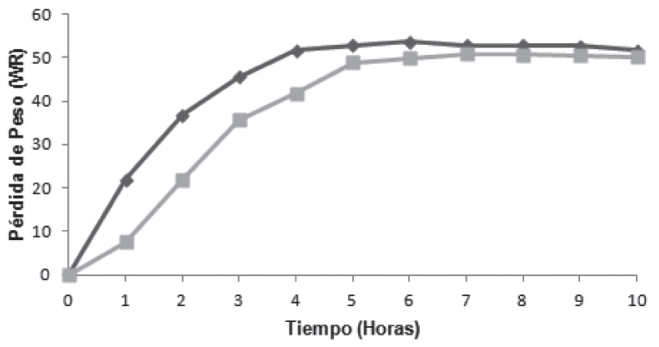

a)

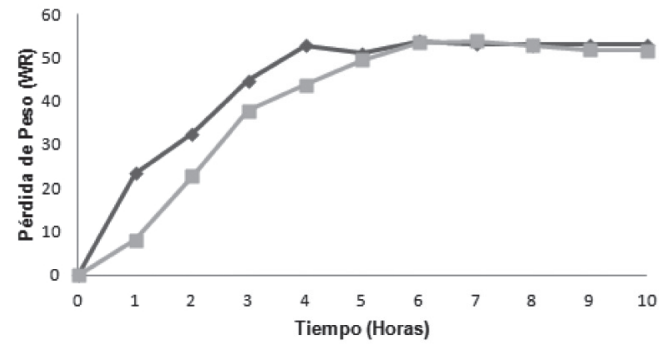

b)

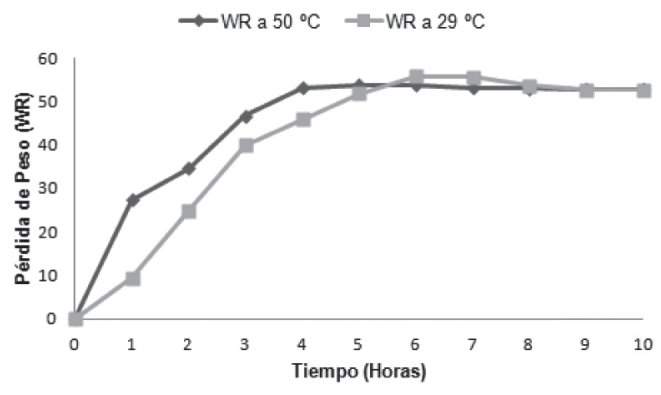

c)

Figura 2. Pérdida de peso (WR) a diferente concentración y temperatura: a) $30^{\circ}$ Brix, b) $40^{\circ}$ Brix, c) $60{ }^{\circ}$ Brix. 


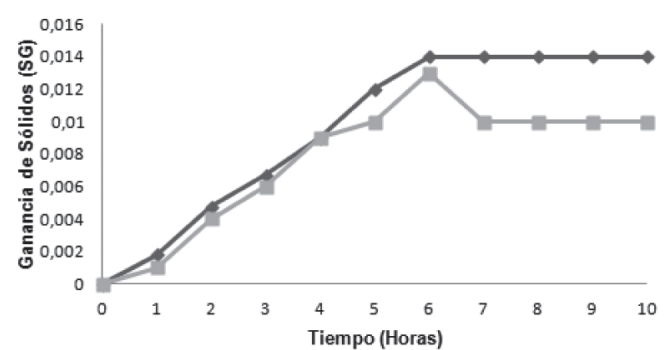

a)

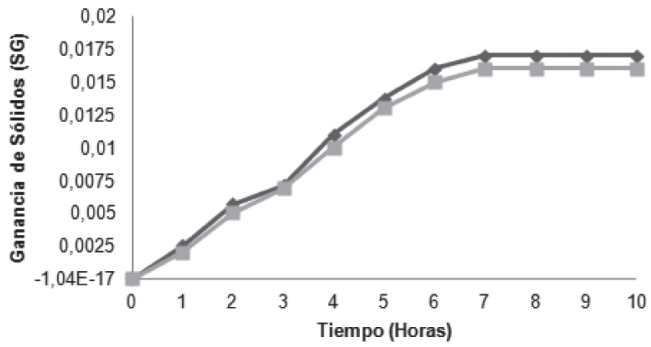

b)

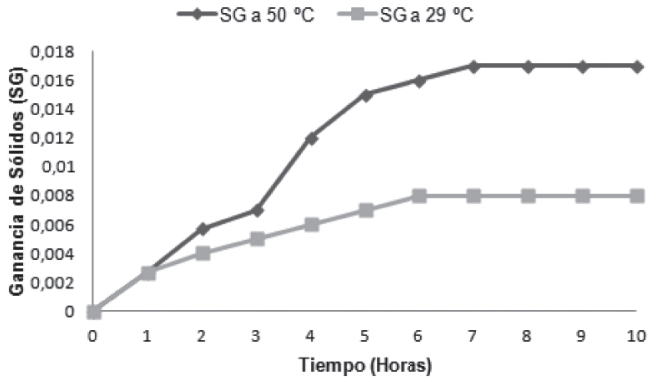

c)

Figura 3. Ganancia de sólidos (SG) a diferente concentración y temperatura: a) $30^{\circ}$ Brix, b) $40{ }^{\circ}$ Brix, c) $60{ }^{\circ}$ Brix.

et al. 2003) concluyeron que soluciones concentradas, por ser más viscosas, obstaculizan el paso de los solutos que se hallan en la solución, al desarrollarse una capa de soluto sobre el alimentos. Este fenómeno, se conoce como impregnación.

Moreira \& Xidieh, (2004), quienes obtuvieron resultados similares, indican que la elección de un medio de alta concentración, trae algunos beneficios en términos de pérdida de agua más rápido; sin embargo, se logra una ganancia mucho mayor de sólidos.

En general, entre las cuatro y cinco horas de proceso, se alcanzaron las mayores tasas de deshidratación, lo cual, se podría tener en cuenta a la hora de aplicar procesos de deshidratación osmótica de pulpa de tamarindo.

Índice de efectividad. Valores altos indican que el proceso osmótico es más efectivo, debido a que WL es mayor que SG (Ayala et al. 2010). En la figura 4, se observa que el índice de efectividad al inicio del proceso está más influenciado por las condiciones de temperatura, siendo mayor para los tratamientos con la aplicación de temperaturas de $50^{\circ} \mathrm{C}$. A medida que avanza el proceso de osmodeshidratación, algunos valores de índices de efectividad presentaron valores cercanos entre los tratamientos y con tendencia a la disminución, lo que puede ser un indicio de la disminución en las velocidades de pérdida de agua o ganancia de solutos (Ayala et al. 2010; Chenlo et al. 2006). Al final del proceso osmótico, se evidenció que este parámetro presenta los valores más significativos para el tratamiento con mayor temperatura y concentración.

Análisis estadístico. Los resultados del ANOVA muestran que la concentración, la temperatura y el tiempo con P-value menores a 0,001 son altamente significativos para la pérdida de agua, pérdida de peso y ganancia de sólidos en la osmodeshidratación de pulpa de tamarindo, tal como lo indican la tabla 1.

Para un mejor análisis del proceso y del diseño experimental, se realizaron interacciones entre los factores: concentración (A), temperatura $(\mathrm{B})$ y tiempo $(\mathrm{C})$, observándose en la tabla ANOVA que las interacciones $A B, A C, B C$ y $A B C$ tienen una diferencia altamente significativa, todos con un P-value menor a 0,001 (Tabla 1). Esto quiere decir, que la pérdida de agua, pérdida de peso y ganancia de sólidos en el proceso de deshidratación osmótica de pulpa de tamarindo se ve influenciado por la temperatura, la concentración, el tiempo y sus interacciones.

Conflictos de intereses: El manuscrito fue preparado y revisado con la participación de todos los autores, quienes declaramos que no existe ningún conflicto de intereses que ponga en riesgo la validez de los resultados presentados. 
Tabla 1. Análisis de varianza para pérdida de agua, pérdida de peso y ganancia de sólidos.

\begin{tabular}{|c|c|c|c|c|c|}
\hline \multicolumn{6}{|c|}{ Pérdida de Agua } \\
\hline Fuente & Suma de Cuadrados & Gl & Cuadrado Medio & Razón-F & Valor-P \\
\hline \multicolumn{6}{|l|}{ EFECTOS PRINCIPALES } \\
\hline A:Concentración & 146,846 & 2 & 73,423 & 131,39 & 0,0000 \\
\hline B:Temperatura & 1140,65 & 1 & 1140,65 & 2041,23 & 0,0000 \\
\hline C:Tiempo & 26229,7 & 9 & 2914,41 & 5215,40 & 0,0000 \\
\hline \multicolumn{6}{|l|}{ INTERACCIONES } \\
\hline$A B$ & 42,7309 & 2 & 21,3654 & 38,23 & 0,0000 \\
\hline$A C$ & 49,7125 & 18 & 2,76181 & 4,94 & 0,0000 \\
\hline $\mathrm{BC}$ & 1269,14 & 9 & 141,016 & 252,35 & 0,0000 \\
\hline $\mathrm{ABC}$ & 63,4417 & 18 & 3,52454 & 6,31 & 0,0000 \\
\hline RESIDUOS & 67,057 & 120 & 0,558808 & & \\
\hline TOTAL (CORREGIDO) & 29009,3 & 179 & & & \\
\hline \multicolumn{6}{|c|}{ Pérdida de Peso } \\
\hline \multicolumn{6}{|l|}{ EFECTOS PRINCIPALES } \\
\hline A:Concentración & 289,122 & 2 & 144,561 & 457,84 & 0,0000 \\
\hline B:Temperatura & 987,668 & 1 & 987,668 & 3128,08 & 0,0000 \\
\hline C:Tiempo & 23628,0 & 9 & 2625,34 & 8314,81 & 0,0000 \\
\hline \multicolumn{6}{|l|}{ INTERACCIONES } \\
\hline$A B$ & 152,556 & 2 & 76,2779 & 241,58 & 0,0000 \\
\hline$A C$ & 171,875 & 18 & 9,54862 & 30,24 & 0,0000 \\
\hline $\mathrm{BC}$ & 1175,44 & 9 & 130,604 & 413,64 & 0,0000 \\
\hline $\mathrm{ABC}$ & 596,967 & 18 & 33,1648 & 105,04 & 0,0000 \\
\hline RESIDUOS & 37,8891 & 120 & 0,315742 & & \\
\hline TOTAL (CORREGIDO) & 27039,5 & 179 & & & \\
\hline \multicolumn{6}{|c|}{ Ganancia de Sólidos } \\
\hline \multicolumn{6}{|l|}{ EFECTOS PRINCIPALES } \\
\hline A:Concentración & 0,00170497 & 2 & 0,000852486 & 262,85 & 0,0000 \\
\hline B:Temperatura & 0,000259921 & 1 & 0,000259921 & 80,14 & 0,0000 \\
\hline C:Tiempo & 0,001685 & 9 & 0,000187222 & 57,73 & 0,0000 \\
\hline \multicolumn{6}{|l|}{ INTERACCIONES } \\
\hline $\mathrm{AB}$ & 0,0000706403 & 2 & 0,0000353202 & 10,89 & 0,0000 \\
\hline$A C$ & 0,000823352 & 18 & 0,0000457418 & 14,10 & 0,0000 \\
\hline $\mathrm{BC}$ & 0,00018175 & 9 & 0,0000201945 & 6,23 & 0,0000 \\
\hline $\mathrm{ABC}$ & 0,000174791 & 18 & 0,0000097106 & 2,99 & 0,0002 \\
\hline RESIDUOS & 0,000389187 & 120 & 0,0000032432 & & \\
\hline TOTAL (CORREGIDO) & 0,00528961 & 179 & & & \\
\hline
\end{tabular}

Todas las razones-F se basan en el cuadrado medio del error residual. 


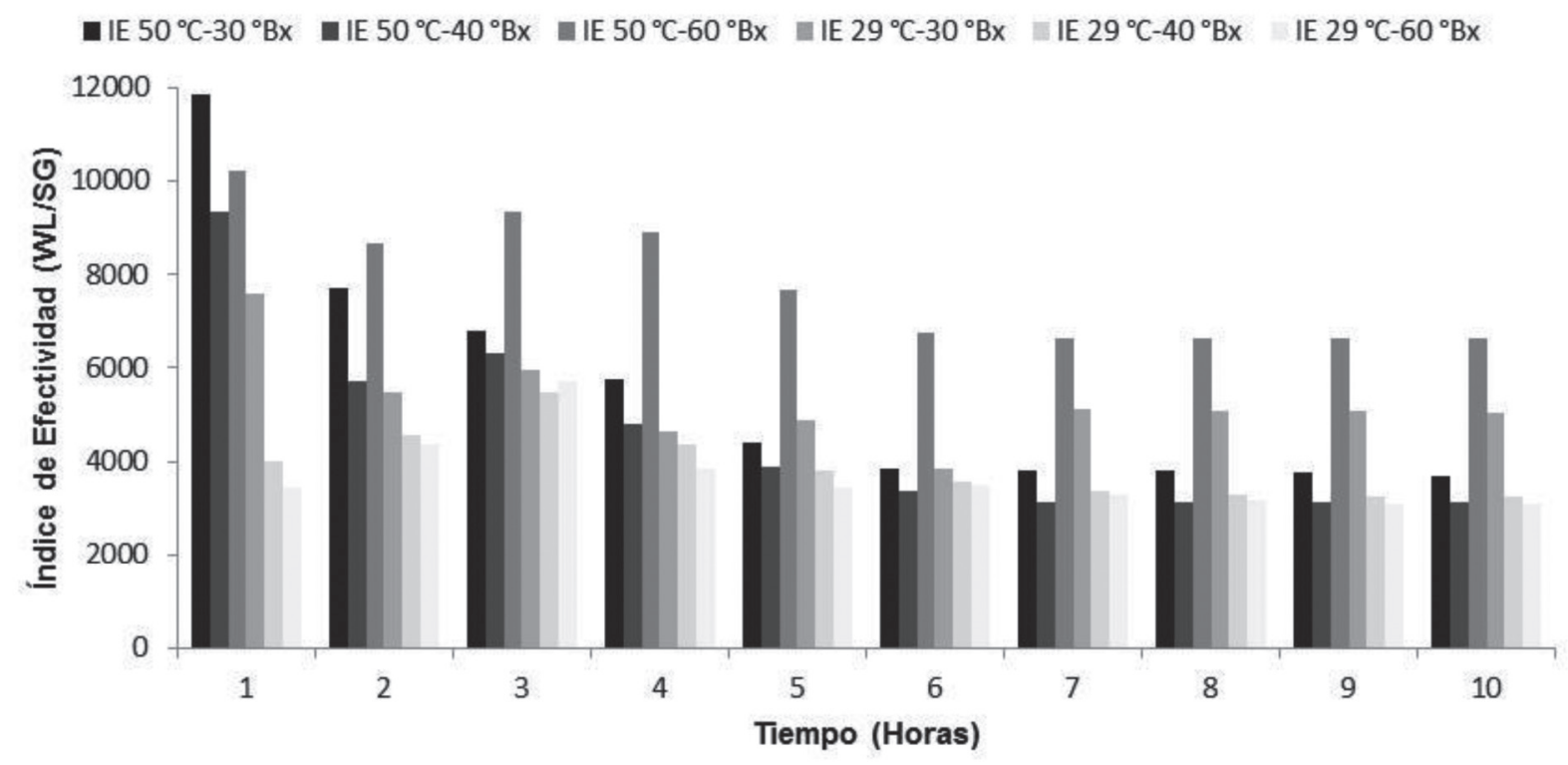

Figura 4. Variación del índice de efectividad (IE) de DO de pulpa de tamarindo.

\section{BIBLIOGRAFÍA}

1. AGNELLI, M.; MARANI, C.; MASCHERONI, R. 2005. Modelling of heat and mass transfer during (osmo) dehydrofreezing of fruits. J. Food Eng. (United States). 69(4):415-424.

2. AHMED, J.; RAMASWAMY, H.S.; SASHIDHAR, K.C. 2007. Rheological characteristics of tamarind (Tamarindus indica L.) juice concentrates. LWT-Food Sci.Techn. (Suiza). 40(2):225-231.

3. AOAC. 2005. Official Methods of Analysis of the AOAC International. 18th ed. Horowitz, W.; Latimer Jr., G.W. (eds) Association of Analytical Chemists. Maryland (United States). 780p.

4. AYALA, A.A.; GIRALDO, C.J.; SERNA, L. 2010. Cinéticas de deshidratación osmótica de pitahaya amarilla (Selenicereus megalanthus). Interciencia. (Venezuela). 35(7): 539-544.

5. CATAldo, A.; CANNAZZA, G.; DE BENEDETTO, E.; SEVERINI, C.; DEROSSI, A. 2011. An alternative method for the industrial monitoring of osmotic solution during dehydration of fruit and vegetables: A test-case for tomatoes. J. Food Eng. (United States). 105:186-192.
6. CHENLO, F.; CHAGURI, L.; SANTOS, F.; MOREIRA, R. 2006. Osmoticdehydration/impregnation kineticsof padrón pepper (Capsicum annuumL. Longum) with sodium chlor ide solutions:process modelling andcolour analysis. FoodSci. Technol. Int. 12:221-227.

7. DELLA ROCCA, P.; MASCHERONI, R. 2011. Deshidratación de papas por métodos combinados de secado: deshidratación osmótica, secado por microondas y convección con aire caliente. Proyecciones. (Argentina). 9(2):11-26.

8. DERMESONLOUOGLOU, E.K.; POURGOURI, S.; TAOUKIS, P.S. 2008. Kinetic study of the effect of the osmotic dehydration pre-treatment to the shelf life of frozen cucumber.Innovative Food Sci. Emerging Technol.. 9: 542-549.

9. GARCÍA, A.; MUÑIZ, S.; HERNÁNDEZ, A.; GONZÁLEZ, L.M.; FERNÁNDEZ, D. 2013. Análisis comparativo de la cinética de deshidratación Osmótica y por Flujo de Aire Caliente de la Piña (Ananas comosus, variedad Cayena lisa). Postcosecha: Biología y Tecnología. (Cuba). 22(1):62-69.

10. GIRALDO, D.P.; ARANGO, L.M.; MÁRQUEZ, C.J. 2004. Osmodeshidratación de mora de castilla (Rubus glaucus Benth) con tres agentes edulcorantes. Rev. Fac. Nal. Agr. (Colombia). 57(1):2257-2274. 
11. KOWALSKA, H.; LENART, A. 2001. Mass exchange during osmotic pretreatment of vegetables. J. Food Eng. 49(2-3):137-140.

12. LAZARIDES, H.N.; KATSANIDIS, E.; NICKOLAIDIS, A. 1995. Mass transfer during osmotic preconcentration aiming at minimal solid uptake. J. Food Eng. 25(2):151-166.

13. MOREIRA, P.; XIDIEH, F. 2004. Mass transfer kinetics of osmotic dehydration of cherry tomato. J. Food Eng. 61(3):291-295.

14. MÚJICA-PAZ, H.; VALDEZ-FRAGOSO, A.; LÓPEZ-MALO, A.; PALOU, E.; WELTI-CHANES, W. 2003. Impregnation and osmotic dehydration of some fruits: effect of the vacuum pressure and syrup concentration. J. Food Eng. 57(4):305-314.

15. NTC. 440. Norma Técnica Colombiana para métodos de ensayo de productos alimenticios. Primera edición. Bogotá (Colombia). 4p.

16. OROZCO-SANTOS, M. 2001. El cultivo de tamarindo (Tamarindus indica L.) en el trópico seco de México. Folleto Técnico Nº1. Ed. Tecomán, Col. (México). 96p.

17. PALOU, E.; LÓPEZ-MALO, A.; ARGAIZ, A.; WELTI, J. 1994. The use of Peleg's equation to model osmotic concentration of papaya. Drying Techn. (United States). 12(4):965-978.

18. PARK, J.K.; BIN, A.; BROD, F.P.R.; PARK, T.H.K.B. 2002. Osmotic dehydration kinetics of pear D'anjou (PyruscommunisL.). J. Food Eng. 52(3):293-298.

19. SALVATORI, D.; ANDRES, A.; CHIRALT, A.; FITO, P. 1999. Osmotic dehydration progression in apple tissue I: spatial distribution of solutes and moisture content. J. Food Eng. 42(3):125-132.

20. SILVA, M.A.C.; ERNESTO DA SILVA, Z.; COCCO, V.; DARCHE, S. 2012. Mass transfer during the osmotic dehydration of West Indian cherry.LWT - Food Sci. Technol. 45:246-252.

21. SPIAZZI, E.; MASCHERONI, R. 1997. Mass transfer model for osmotic dehydration of fruits and vegetables-I. Development of the simulation model. J. Food Eng. 34(4):387-410.

22. TOĞRUL, I.T.; ÍSPIR. A. 2008. Equilibrium distribution coefficients during osmotic dehydration of apricot. Food Bioprod. Proc. (Europa). 86:254-267.

Recibido: Mayo 20 de 2013.

Aceptado: Enero 20 de 2014

Como citar:

Acevedo, D.; Tirado, D., Guzmán, L. 2014. Deshidratación osmótica de pulpa de tamarindo (Tamarindus indica L.): influencia de la temperatura y la concentración. Rev. U.D.C.A Act. \& Div. Cient. 17(1): 123-130. 